\title{
Energetic Particles in the Local Bubble
}

\author{
G.E. Morfill and M.J. Freyberg \\ Max-Planck-Institut für extraterrestrische Physik, D-85740 Garching, Germany
}

\begin{abstract}
The current status of observations of energetic particles in the "local bubble" is reviewed. This includes primarily "direct" measurements of cosmic rays made in the Solar System, but also the "remote sensing" made possible by observing cosmic ray produced $\gamma$-rays in the nearby interstellar clouds. Since the energetic events responsible for the formation of our local bubble may also have produced copious amounts of cosmic rays, fossil records are examined to determine whether there is a corresponding signature. The observations show that: 1) the cosmic ray (proton) intensity is fairly homogeneous throughout the local bubble and its adjacent interstellar clouds, 2) there is some evidence for a "recent" local cosmic ray injection about 40,000 years ago, 3) on longer time scales (a few million years) the cosmic ray intensity was constant within a factor two, 4) there is apparently some "activity" in the Orion cloud, as evidenced by low energy $\gamma$-ray signatures, and 5) there are two unexplained observations - the variations in the energy spectra, in particular the significantly flatter spectrum of heavy cosmic rays ( $\mathrm{Fe}$ ) and the matter path length variation, which yields consistently larger path lengths for the lighter elements $(\mathrm{H}, \mathrm{He})$. It is suggested that these observations are compatible with two cosmic ray populations - an older one in equilibrium with losses from the galaxy and a younger one which is not yet strongly affected by losses. The latter could be a cosmic ray signature of the formation of the local bubble.
\end{abstract}

\section{Introduction}

Our "local bubble" is a somewhat complicated and inhomogeneous region in the galactic disk, which was probably formed a few million years ago through a series of supernova explosions, probably originating in the ScorpiusCentaurus Association (see Fig.1 of Frisch in this volume and the reviews by Breitschwerdt et al. 1996, Egger et al. 1996, and Frisch 1995). The total amount of energy liberated in such an event (or series of events) is of the order $10^{52} \mathrm{erg}$. Supernovae are believed to be the most important sources of cosmic rays (CRs) - at least for energies below about $10^{5} \mathrm{GeV}$ - with a few percent of the available energy "channelled" into energetic particles. The physical process believed to be responsible is "diffusive shock acceleration" (e.g. Axford et al. 1977, Krymsky 1977, Bell 1978a, b, Drury 1983, Völk et al. 1981, Morfill et al. 1984, Lagage \& Cesarsky 1983, Ellison et al. 1981 for the early treatment of different aspects such as wave production, damping, energy losses, time dependence, the injection processes, maximum energy etc.).

The signature of these energetic events and the associated processes that marked the formation of the local bubble might still be present in CRs. Let 
us illustrate this with some numbers: If the total amount of energy in CRs is $E_{\mathrm{CR}} \sim 3 \times 10^{50} \mathrm{erg}$ ( $3 \%$ of our assumed total energy), if the mean time of local bubble formation occurred $t_{\mathrm{B}} \sim 3 \times 10^{6}$ years ago and if the current size of the bubble is $R_{\mathrm{B}} \sim 200 \mathrm{pc}$ in diameter, then the CRs produced in the many supernova shock waves can fill this cavity completely, provided the diffusion coefficient is $\kappa>R_{\mathrm{B}}^{2} / t_{\mathrm{B}}=10^{27} \mathrm{~cm}^{2} \mathrm{~s}^{-1}$. The CR energy density in the local bubble is then $\epsilon_{\mathrm{CR}}=3 E_{\mathrm{CR}} / 4 \pi R_{\mathrm{B}}^{3}=3 \times 10^{12} \mathrm{erg} \mathrm{cm}^{-3}-$ or less, if the CRs have diffused further than $R_{\mathrm{B}}$. The obvious signature to look for is a local CR enhancement.

The energy spectrum of CRs is also characteristic. In diffusive shock acceleration one expects a power law with an exponent of about $E^{-2}$ (strong shocks) or $E^{-2.3}$ (mixture of strong and weak shocks - see Bogdan and Völk, 1985). If the diffusion coefficient is energy dependent ( $\kappa$ increases with energy) and there is escape from our galaxy, then the high energy CRs are lost faster and the spectrum steepens some more. There is also a maximum energy (of about $10^{6} \mathrm{GeV}$ ) that can be reached. This is due to a combination of the weakening of propagating shocks and the available time (see Forman \& Morfill 1979, Morfill et al. 1984, Lagage \& Cesarsky 1983). Thus the cut-off signature at $10^{6} \mathrm{GeV}$ should also be looked for. This then characterises the expected CR population in the vicinity of our solar system. If there were no other, more recent, CR sources in our local bubble, the CR intensity would be expected to be fairly uniform throughout. If there is also an older CR population, mixed and diffused over a correspondingly larger volume, then the "local bubble component" referred to above will simply be added on top of this older component.

The CR signature of the bubble forming "event" may be observable in other ways, too, e.g. in the traversed matter path length distribution (if there are young and old CR populations) and in time variations of fossil records. These may provide additional clues to the ones discussed above, i.e. the medium scale spatial CR intensity variation and the energy spectrum. These, therefore, are the CR signatures of interest, and the purpose of this review is to collect and analyse the available information.

\section{Measurements in the Solar System}

CRs have been studied using ground based and spacecraft detectors for many decades now. Below we summarize the most important findings:

In spite of being highly suprathermal with a mean energy in the GeV/ nucleon region (compared to the interstellar gas with a mean particle energy bracketing $\sim 0.001 \mathrm{eV}$ in the case of clouds, $\sim 1 \mathrm{eV}$ in the case of $\mathrm{HI}$ regions and $\sim 1 \mathrm{keV}$ in the case of supernova remnants), CRs nonetheless have roughly the same energy density as their "thermal" counterparts, i.e. $\sim 1.6 \times 10^{-12} \mathrm{erg} \mathrm{cm}^{-3}$. This implies that energetically and dynamically they form an important component of the interstellar medium. 
The highest energy CR particles measured so far (using extensive airshower techniques) have several $10^{20} \mathrm{eV}$ - this is equivalent to a BMW 735 moving at $1 \mathrm{~km} /$ hour (if you owned such a car you would never travel at this speed, of course, but it is theoretically possible).

The element abundance of CRs is roughly "cosmic", a strong indication that these particles originate from the interstellar medium and not some special source, which may have different abundances. Departures from the cosmic abundance occur, e.g.: $\mathrm{Li}, \mathrm{Be}, \mathrm{B}$ are enhanced by several orders of magnitude, as are the elements with atomic numbers $20-25$, i.e. just below the Fe-group. This is readily explained by spallation reactions from $\mathrm{C}, \mathrm{N}, \mathrm{O}$ in the former and from $\mathrm{Fe}$ in the latter case.

The CR energy spectrum is a remarkable power law. From about $1 \mathrm{GeV}$ per nucleon to about $10^{6} \mathrm{GeV} \mathrm{n}^{-1}$ the spectral exponent is $E^{-2.75}$, above that until about $10^{9}-10^{10} \mathrm{GeV} \mathrm{n}^{-1}$ it steepens to $E^{-3.0}$. The turn-up of the helium flux below $\sim 60 \mathrm{MeVn}^{-1}$ is due to the additional flux of the anomalous ${ }^{4} \mathrm{He}$ component. The Fe-spectrum is harder than that for $\mathrm{H}$ and $\mathrm{He}$ (cf. Longair 1992). Further measurements in the experimentally difficult energy range from $10^{3}-10^{7} \mathrm{GeV} \mathrm{n}^{-1}$ (Longair 1992) show that "heavy" $\mathrm{CR}$ particles, such as $\mathrm{Fe}$, have similar fluxes as the "light" particles (H, He) above $\sim 10^{4} \mathrm{GeV} \mathrm{n}^{-1}$ (or $\sim 10^{5} \mathrm{GeV} /$ particle). Airshower simulations show in addition, that above $\sim 10^{9} \mathrm{GeV} \mathrm{n}^{-1}$ (or $\sim 10^{10} \mathrm{GeV} /$ particle) protons dominate again. The most likely explanation for this is photodisintegration of these ultra-high-energy particles by interactions with blue-shifted (in the particle's frame) photons from the cosmic microwave background.

The "age" of CRs can be determined in two ways. The most direct is to measure the abundances of radioactive isotopes, a second possibility is provided by the ratio of secondaries to primaries. Secondaries are, for instance, all those particles produced by spallation. Some of these are stable, so their abundances give a measure of the amount of material traversed by the CRs. Given an average density for the interstellar medium, it is then possible to determine the CR "age". However, uncertainties arise, because the volume occupied by CRs (i.e. the extent of the galactic halo) is not known too well. Basically, it is not clear how much time CRs spend on average in denser portions of the interstellar medium (e.g. the galactic disk) and how much time in the more tenuous regions (e.g. the halo). The amount of material traversed by CRs decreases with increasing energy. This indicates that energetic particles escape faster from the galaxy, i.e. the diffusion mean free path increases with energy. Combining secondary/primary ratios with radioactive isotope abundance measurements (in particular the ${ }^{10} \mathrm{Be} /{ }^{9} \mathrm{Be}$ ratio - see Simpson \& Garcia-Munoz, 1988) gives a mean CR "age" of $\tau_{\mathrm{CR}} \sim 10^{7}$ years in the GeV-range. Compared to the age of our galaxy CRs are "young", compared to the age of the local bubble CRs are somewhat older or perhaps even contemporaries. 
There are two possible interpretations for "young" CRs: 1) the production processes are a fairly recent phenomenon of galactic evolution, or 2) CRs have always been produced, but have a finite "life-time" - either getting "destroyed" (e.g. by energy degradation) or escaping from our galaxy. If CRs were destroyed by collisions with the interstellar gas, the secondary abundances would show this. In fact, the deduced path length distribution is exponential, with a mean value of only about $6 \mathrm{~g} \mathrm{~cm}^{-2}$, much less than the $\sim 70 \mathrm{~g} \mathrm{~cm}^{-2}$ required to significantly degrade relativistic CRs. Observed "anomalies" in the path length distribution of secondaries have so far not been interpreted as being connected to the local bubble (see e.g. Morfill et al. 1985 for a discussion).

Direct "fossil" evidence for CR existence can be obtained from tree rings (1000's years), from drill core measurements from the layerings of the polar ice caps (million years) and from meteorites (billion years). The result is that CRs have existed throughout time at roughly the same intensity. The enhancement expected in connection with the local bubble formation is not observed, at least not to the accuracy of the measurements ( $\sim$ factor 2 , see e.g. the reviews in Sonett et al. 1991), however, a more recent enhancement dated about 40,000 years ago (Sonett et al. 1987) suggests a "late" supernova, which may have exploded $\sim 10^{5}$ years ago. The enhancement marks the passage of the supernova shock wave across the solar system in this interpretation.

The conclusion is that CRs have been continuously produced in our galaxy for billions of years and escape into the intergalactic medium in a time scale of $\sim 10^{7}$ years. The implications for the local bubble will be discussed later.

\section{Remote Sensing}

We mentioned earlier that CRs interact with the interstellar gas. In these reactions $\pi^{0}$-mesons may be produced, which subsequently decay into $\gamma$-rays. Of course, there will be other $\gamma$-ray producing reactions (e.g. inverse Compton or Bremsstrahlung - see Fig.1), but it is nevertheless possible to isolate an energy window in $\gamma$-ray emission, from about $10^{2}-10^{4} \mathrm{MeV}$, where the $\pi^{0}$ decay dominates and where the $\gamma$-ray emission can therefore be used as a tracer of CR intensity.

A surprising conclusion from the analysis of the EGRET data (Strong et al. 1997) is the fact that the galactic $\gamma$-ray emission at high energies is significantly larger than that predicted using the local CR spectrum. In fact, a spectrum $\sim E^{-2.4}$ to $E^{-2.5}$ appears to be required for the galaxy as a whole. As we will discuss later, the CR spectrum can show local variations, especially if injection is impulsive, so that this observation may be a pointer to a local signature. For present purposes we are particularly interested in the emission from local clouds at the edge of our local bubble in addition to the global picture. Three pertinent investigations have been performed so far. 


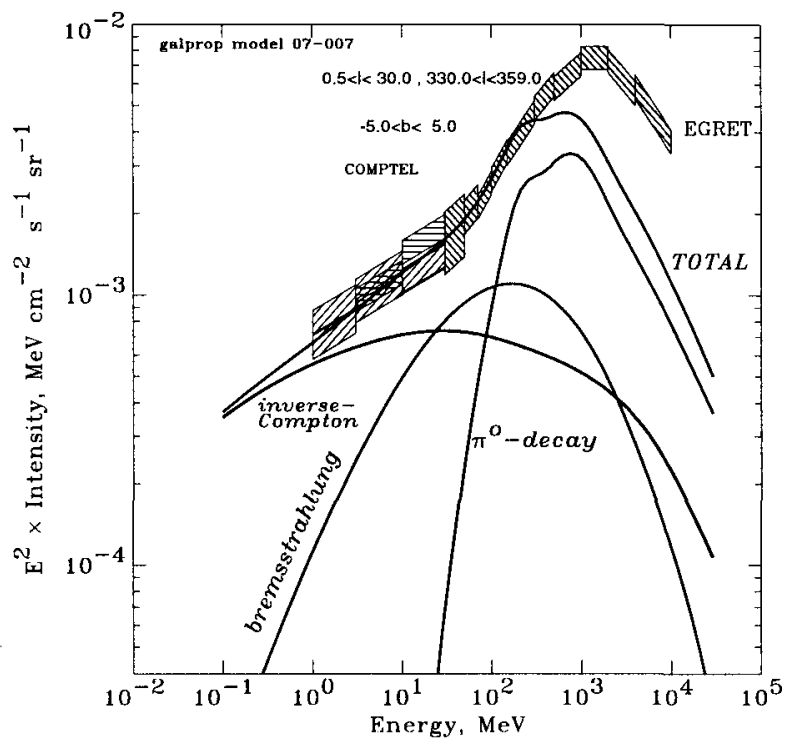

Fig. 1. The computed galactic $\gamma$-ray intensity for various processes and its comparison with experimental data (from Strong et al. 1997).

\subsection{Ophiuchus}

The Ophiuchus molecular cloud has long been regarded as a region of enhanced $\gamma$-ray - and therefore CR - activity. Excesses up to an order of magnitude above the local (solar, in situ) value were quoted. Careful analysis with the more sensitive EGRET instrument has shown that this enhancement is real, but that it is most likely caused by the quasar PKS 1622-253, which is located 20' away from the maximum likelihood position of the formerly identified Ophiuchus source (Hunter et al. 1994). The quasar signature can be identified fairly unambiguously because it is time variable. There is also, possibly, another (as yet unidentified) source, GRO J1631-27 in the vicinity. The resultant $\gamma$-ray production function in Ophiuchus is shown in Fig.2. Removal of the point sources increases the errors in the determination of the cloud's emission, of course.

\subsection{Orion}

The giant cloud complex in Orion was identified as a $\gamma$-ray source some years ago (Caraveo et al. 1980). Recent observations using EGRET were able to resolve both the Orion A and B clouds (Digel et al. 1995). The CO emission contours were compared with the $\gamma$-ray map of the same sky region. There was even a hint in the data that Mon R2 has been detected, although at a lower confidence level than the Orion clouds. The corresponding $\gamma$-ray emissivity 

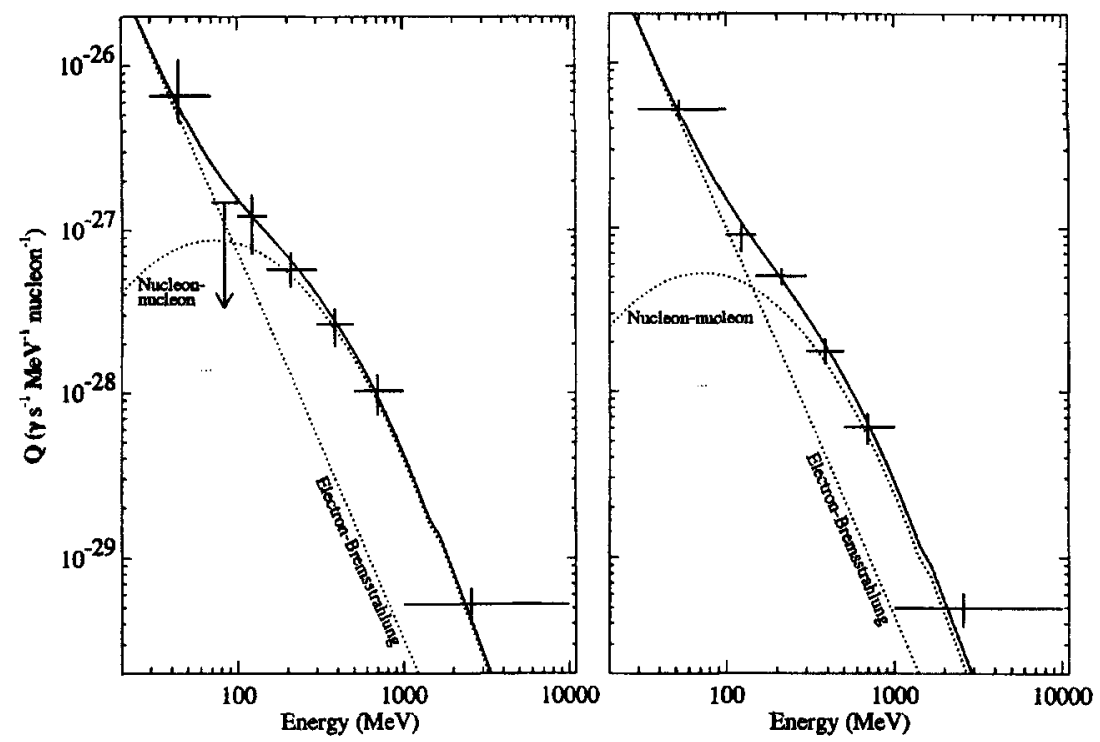

Fig. 2. Left: $\gamma$-ray production function in Ophiuchus derived from the fits to the separate energy ranges on the assumption of an energy spectral index of -2 . The ranges are indicated by the horizontal error bars. The solid curve represents the best-fitting linear combination of the nucleon-nucleon and electron-bremsstrahlung production function used by Bertsch et al. (1993). The individual production functions are also shown as dotted curves (from Hunter et al. 1994).

Right: $\gamma$-ray production function in Orion (from Digel et al. 1995).

is shown in Fig.2. An excess in the $3-7 \mathrm{MeV} \gamma$-ray emission from Orion has been observed with COMPTEL (Bloemen et al. 1997, Bykov et al. 1996). One explanation is that this emission is due to Doppler-broadened $\mathrm{C}$ and $\mathrm{O}$ de-excitation lines, which occur at 4.44 and $6.13 \mathrm{MeV}$. A large flux of low energy (10's MeV) CRs is required. The interaction of such low energy CRs with the cloud would lead to ionisation, knock-on electrons and an X-ray bremsstrahlung flux that should be measurable. The observations performed by ROSAT show only marginally the required X-ray emission (cf. Dogiel et al. 1997, Schönfelder et al. 1997, and references therein).

\subsection{Cepheus and Polaris Flare}

Digel et al. (1996) examined the region $l^{I I}=100^{\circ}$ to $130^{\circ}$ and $b^{I I}=-5^{\circ}$ to $+32^{\circ}$, which contains the Cepheus and Polaris Flares, cloud complexes clearly visible in $\mathrm{CO}$ emission. In addition they also examined the Perseus region. Utilising these new results as well as older studies, we have compiled two tables. Table 1 gives the $\gamma$-ray emissivity above $100 \mathrm{MeV}$. This should be directly proportional to the CR intensity at the location (cloud) where the 
Table 1. $\gamma$-ray emissivity (in $10^{-26} \mathrm{~s}^{-1} \mathrm{sr}^{-1}$ )

\begin{tabular}{c|c|c}
\hline Local & (Strong et al. 1988) & 1.8 \\
Ophiuchus & (Hunter et al. 1994) & $(2.4 \pm 0.2)$ \\
Orion & (Digel et al. 1995) & $(1.7 \pm 0.1)$ \\
Cepheus \& Polaris Flares & (Digel et al. 1996) & $(1.8 \pm 0.1)$
\end{tabular}

measurement was made. These results therefore allow us to "remotely sense" the $\mathrm{CR}$ intensity at specific locations in a volume of radius $\sim 500 \mathrm{pc}$. An important quantity to know - in order to derive the $\gamma$-ray emissivities - is the conversion factor between $\mathrm{CO}$ emission $\left(\mathrm{K} \mathrm{km} \mathrm{s}^{-1}\right)$ and the hydrogen column density $N_{\mathrm{H}}\left(\mathrm{H}\right.$-mols cm$\left.{ }^{-2}\right)$. With the $\gamma$-ray emissivity, $\epsilon_{\gamma}$ (photons $\mathrm{MeV}^{-1}$ $\mathrm{s}^{-1} \mathrm{n}^{-1}$ ), the measured differential $\gamma$-ray flux of a cloud of area $A$ at distance $r$ is $F_{\gamma}=A N_{\mathrm{H}} \epsilon_{\gamma} / 4 \pi r^{2}$ (photons $\mathrm{cm}^{-2} \mathrm{sr}^{-1} \mathrm{~s}^{-1} \mathrm{MeV}^{-1}$ ).

Table 2. Hydrogen column/CO column (units: $10^{20} \mathrm{H}$-mols $\mathrm{cm}^{-2} /\left(\mathrm{K} \mathrm{km} \mathrm{s}^{-1}\right)$ )

\begin{tabular}{c|c|c}
\hline Ophiuchus & (Hunter et al. 1994) & $(1.1 \pm 0.2)$ \\
Orion & (Digel et al. 1995) & $(1.06 \pm 0.14)$ \\
Cepheus \& Polaris Flares & (Digel et al. 1996) & $(0.92 \pm 0.14)$ \\
Perseus & (Digel et al. 1996) & $(2.5 \pm 0.9$ ) \\
Galactic Average & (Bertsch et al. 1993) & 1.6 \\
High Latitude Cirrus & (Heithausen \& Thaddeus 1990) & 0.5
\end{tabular}

Since the CO emission of interstellar clouds is readily measurable, $\epsilon_{\gamma}$ can be determined (in principle) once the conversion factor is known. Various results are summarised in Table 2. Table 1 suggests a fairly uniform CR intensity, at least in a volume of $0.5 \mathrm{kpc}$ or less, with possibly just the hint of an enhancement remaining at $\rho$-Ophiuchus. The quality of this statement is enhanced by the results compiled in Table 2 .

\section{Summary, Conclusions, and Speculations}

In terms of the possible "cosmic ray signatures" of the processes that led to the formation of the local bubble, the available measurements to date give the following "answers":

1. Local Enhancement of Cosmic Rays: No evidence was found from $\gamma$-rays (to within $10 \%$ ) of CR intensity variations in a region around the Sun of radius $\sim 0.5 \mathrm{kpc}$. Based on our earlier estimate of a CR energy injection of $\sim 3 \times 10^{50}$ erg some 3 million years ago, this requires a diffusion coefficient $\kappa>2.5 \times 10^{28} \mathrm{~cm}^{2} \mathrm{~s}^{-1}-$ if the transport really was (is) diffusive. Note that 
this constraint applies to protons only, because they provide most of the $\gamma$ rays.

2. Cosmic Ray Age: The inferred exponential path length distribution also implies an equivalent $C R$ age distribution, with a mean of $\sim 10$ million years. The lack of a clear signature in the path length distribution (a smaller grammage) dating back $\sim 3$ million years could imply that our local bubble formed much earlier (this might not be compatible with the measurements of the local gas), or it implies that the "local" CRs from this event are sufficiently diluted in the galactic background population.

3. Cosmic Ray Spectrum: The observed spectral break occurs approximately at the highest energy computed for acceleration in multiple supernova remnant shocks $\left(10^{6} \mathrm{GeV} /\right.$ particle). This does not constitute a "signature" for our local bubble, however. It could and probably does, mean that shock acceleration is the dominant process for CR production in our galaxy generally, and that the local bubble is nothing special.

4. Time Variation: The identification of a supernova shock wave sweeping across the solar system about 40,000 years ago (Sonett et al. 1987) demonstrates that some kind of fossil records of such events should, and do, exist. The lack of identification of multiple events within a period of $\sim 1$ million years is explained as follows: polar ice cores records until now do not go far enough back in time, and meteoritic data do not yield a sufficiently fine time resolution since they only provide integral measurements. In other words, current fossil techniques cannot be expected to identify the event.

5. The Cosmic Ray Age, and Spectrum - Again: We mentioned earlier that the diffusive shock acceleration process yields a spectrum with a power law exponent of -2 to -2.3 (if weaker shocks are included). Further steepening to the observed value of -2.75 was attributed to energy dependent escape from our galaxy. However, this steepening is only obtained in the steady state $\left(t>\tau_{\mathrm{CR}}\right)$. For impulsive injection, which occurred less than $\sim \tau_{\mathrm{CR}}$ ago, this is different. Obviously there will be some steepening because faster diffusive spreading of the more energetic particles dilutes them more. On the other hand, self-confinement (e.g. due to coupling onto the interstellar medium (e.g. via waves produced as a result of the streaming instability, when the CR energy density exceeds that of the gas - or plasma - by a sufficient amount) is energy independent, and would not affect the spectrum.

Well, there are two puzzling observations regarding $\mathrm{CRs}$, which we have glossed over so far in order to "keep to the major issues". In doing so, we may actually have overlooked what could be a major issue:

The first observation, which we have simply stated as a fact, is the flatter Fe-spectrum, which is again a power law, but varies as $\sim E^{-2.5}$ from $10^{3}-$ $10^{7} \mathrm{GeV} /$ particle. The second observation, which we have not discussed in detail so far, is the observation that the amount of material traversed by CRs decreases as the CR nucleus gets heavier, from about $10 \mathrm{~g} \mathrm{~cm}^{-2}$ for $\mathrm{H}$ to about $3 \mathrm{~g} \mathrm{~cm}^{-2}$ for the iron group. 
So far, all attempts at explaining these observations (see e.g. Morfill et al. 1985 and references therein) have assumed local path length enhancements near the source region. These observations could also be explained using two $\mathrm{CR}$ components - one associated with the formation of the local bubble, the second averaging over many source regions in the galactic disk:

A: The lower matter path length derived for the heavy CRs (Fe) suggests that this is a younger component. Conversely, the greater matter path length derived for the light elements $(\mathrm{H}, \mathrm{He})$ suggests that this is an older component. The flatter spectrum observed for Fe suggests that the heavy CRs are not yet in equilibrium with losses from the galaxy, i.e. they are younger than $10^{7}$ years. Conversely, the steeper spectra observed for the light elements $(\mathrm{H}, \mathrm{He})$ suggest that they are in equilibrium - which again would imply that they are older.

If we accept this "evidence", the question is, how do we account for a young local population of heavy CRs and an older non-local population of light CRs? And how do the GRO $\gamma$-ray data fit into this scheme, since they appear to require a flatter $\mathrm{CR}$ spectrum throughout the galaxy? Maybe the proton spectrum is the anomaly, not the heavies? If we follow this hypothesis, we would come to the following argument:

$\mathrm{B}$ : The equilibrium $\mathrm{CR}$ spectrum throughout the galaxy is a power law with an exponent $\sim E^{-2.5}$. We observe this locally only for the heavy elements, and must therefore conclude that these are "old" and equilibrated with losses. (Note that the $\mathrm{He}, \mathrm{CNO}$ and Ne-Si spectra also appear harder than the proton spectrum, but less hard than the Fe spectrum). The protons have a softer spectrum. This could be a signature of time dependent shock acceleration, where different shocks of different time scales are superposed, each able to accelerate particles to a somewhat different upper energy cut-off, resulting - albeit fortuitously - in a steeper spectrum, or it could signify enhanced local losses.

Hypothesis A leads to the requirement that the local population was simply enhanced with heavy particles. Hypothesis B leads to the requirement that we have a "young" local proton component added on top of the uniform galactic CR component.

It is clear that investigation of local variations in CR production and losses as well as in transport appears necessary to understand the available information from $\gamma$-ray and "in situ" CR measurements. Being located in a local bubble of comparatively recent origin, and having the "remote sensing" capability of $\gamma$-ray measurements, gives us a reasonable chance to unravel the puzzles that observations have presented us with.

Acknowledgements. We wish to acknowledge the help in preparing this manuscript, both through discussions and otherwise, given by many of our colleagues at MPE. In particular, we wish to thank Andy Strong for some detailed information. 


\section{References}

Axford W.I., Leer E., Skadron G., 1978, Proc. $15^{\text {th }}$ Int. Cosmic Ray Conf. 11, 132

Bell A.R., 1978a, MNRAS 182, 147

Bell A.R., 1978b, MNRAS 182, 443

Bertsch D.L. et al., 1993, ApJ 416, 587

Bloemen H. et al., 1997, ApJ 475, L25

Bogdan T., Völk H.J., 1985, A\&A 122, 129

Breitschwerdt D. et al, 1996, Space Sci. Rev. 78, 183

Bykov A.M., Bozhokin S.V., Bloemen H., 1996, A\&A 307, L37

Caraveo P.A. et al., 1980, A\&A 91, L3

Digel S.W., Hunter S.D., Mukherjee R., 1995, ApJ 441, 270

Digel S.W. et al., 1996, ApJ 463, 609

Dogiel V.A., Freyberg M.J., Morfill G.E., Schönfelder V., 1997, Proceedings of the $4^{\text {th }}$ Compton Symposium, in press

Drury L.O'C., 1983, Rep. Progr. Phys. 46, 163

Egger R.J., Freyberg M.J., Morfill G.E., 1996, Space Sci. Rev. 75, 511

Ellison D.C., Jones F.C., Eichler D., 1981, Journal of Geophysics 50, 110

Ellison D.C., Drury L.O'C., Meyer J.-P., 1997, preprint (to appear in ApJ)

Forman M.A., Morfill G.E., 1979, Proc. $16^{\text {th }}$ Int. Cosmic Ray Conf. 2, 70

Frisch P.C., 1995, Space Sci. Rev. 72, 499

Heithausen A., Thaddeus, P., 1990, ApJ 353, L49

Hunter S.D., Digel S.W., de Geus E.J., Kanbach G., 1994, ApJ 436, 216

Krymsky G.F., 1977, Dokl. Akad. Nauk SSR 234, 1306

Kulsrud R.M., Pearce W.D., 1969, ApJ 156, 445

Lagage P.O., Cesarsky C., 1983, A\&A 125, 249

Longair M.S., 1992, High Energy Astrophysics Vol.1, $2^{\text {nd }}$ ed., Cambridge UP

Morfill G.E., Forman M.A., Bignami G., 1984, ApJ 284, 856

Morfill G.E., Meyer P., Lüst R., 1985, ApJ 296, 670

Schönfelder V., Dogiel V.A., Freyberg M.J., Morfill G.E., 1997, these proceedings

Simpson J., 1983, in: Composition and origin of Cosmic Rays, ed. M. Shapiro (Reidel, Dordrecht), 1

Simpson J., Garcia-Munoz M., 1988, Space Sci. Rev. 46, 205

Sonett C.P., Giampapa M., Matthews M. (eds.), 1991, "The Sun in Time", Univ. of Arizona Press, Tucson

Sonett C.P., Morfill G.E., Jokipii J.R., 1987, Nature 330, 458

Strong A.W. et al., 1997, Proceedings of $4^{\text {th }}$ Compton Symposium, in press

Völk H.J., Morfill G.E., Forman M.A., 1981, ApJ 249, 161 